\title{
Acute glaucoma: results of treatment by bilateral simultaneous iridectomy, now without admission to hospital
}

\author{
R. M. INGRAM AND J. R. ENNIS \\ From Kettering and District General Hospital
}

SUMMARY Medical treatment followed by bilateral simultaneous iridectomy appears to be an effective basis for managing a patient who presents with acute glaucoma. Admission to hospital is seldom necessary. There was minimal evidence that delay in starting treatment caused more sight to be lost, but as the patient's age increased the prognosis for recovery of sight in an affected eye decreased quite significantly. The long-term prognosis for the sight of an individual patient who has had prophylactic iridectomy in the unaffected eye is excellent.

For patients presenting with unilateral acute glaucoma many surgeons routinely do a prophylactic iridectomy on the second eye some days after the affected eye has been operated on. However, Lowe ${ }^{1}$ has suggested that prophylactic iridectomy could be performed while the first eye was receiving medical treatment, and he has operated on both eyes simultaneously. Bilateral simultaneous iridectomy was adopted here as a routine policy, after appropriate medical treatment, in December 1970. During the next 10 years 75 patients presented with acute glaucoma, and the results of managing them this way are presented. Thirty-two patients were treated without admission to hospital.

The visual results have been analysed in a way that permits assessment of the effect of the patient's age and the duration of the attack on the acuity recovered.

\section{Patients and methods}

Of 75 patients 52 were female and 23 male. Their ages ranged from 34 to 89 years. Cases of acute glaucoma following retinal vein thrombosis or associated with dislocated lenses were excluded.

The right and left eyes alone were equally frequently affected in 72 patients, and both eyes were simultaneously affected in 3 . In one patient acute glaucoma affected an only eye, and 2 patients had had acute glaucoma in the other eye prior to 1970 and Correspondence to Dr R. M. Ingram, Kettering and District General Hospital, Rothwell Road, Kettering, Northamptonshire NN168UZ. declined prophylactic iridectomy. Thus 78 affected eyes and 69 eyes which had a prophylactic iridectomy are assessed.

\section{TREATMENT REGIMEN}

All patients received acetazolamide $500 \mathrm{mg}$ immediately. It was usually given intramuscularly. A few patients were given it intravenously, but this was stopped when one man had acute retention of urine within 24 hours. Acetazolamide was continued orally in a dosage of $250 \mathrm{mg}$ 6-hourly. Pilocarpine $4 \%$ drops were also instilled hourly or 6-hourly to the affected eye, the frequency depending on the severity of the attack, and 6-hourly to the unaffected eye until operation, some time within the succeeding 4 days.

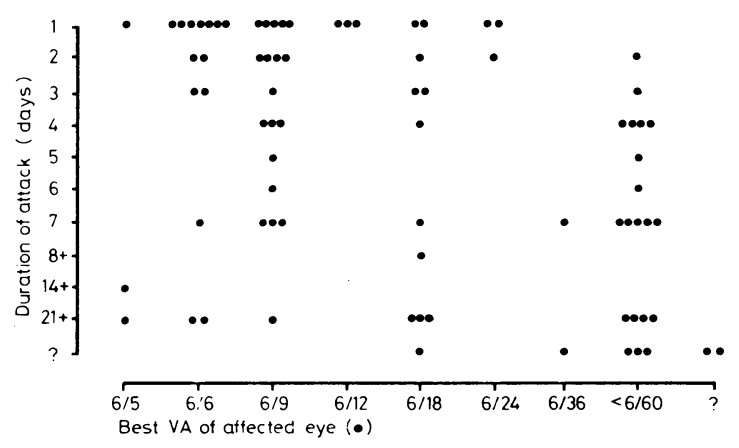

Fig. 1 Duration of symptoms and the best recorded acuity of the affected eye after treatment. 


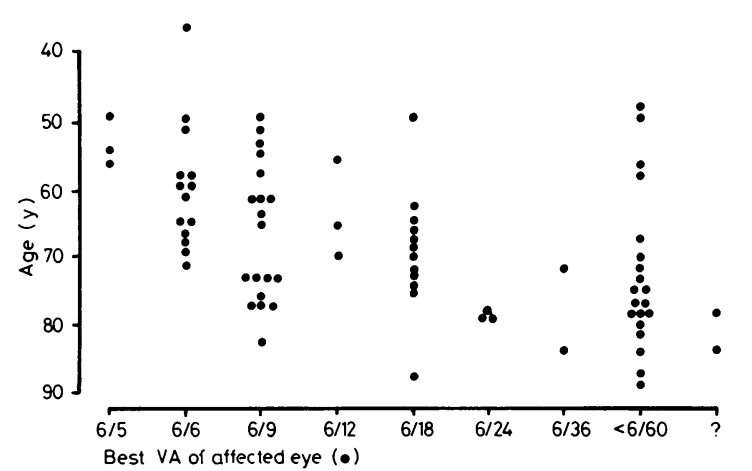

Fig. 2 Age of the patients and the best recorded acuity of the affected eye after treatment.

All these patients were operated upon by the same surgeon and, with one exception, under local anaesthetic. Retrobulbar haemorrhage prevented bilateral simultaneous surgery in 5 cases.

\section{Results}

The number of patients presenting in each year is shown in Table 1, together with their duration of stay in hospital.

Details of the visual results of treatment of both the affected and the unaffected eyes of individual patients can be provided on request to the authors. They have been summarised in Figs. 1-3. Data on 7 patients were incomplete, and these have been omitted from subsequent analysis. These figures have been analysed in a way that permits assessment of the possible effects of the patient's age and/or the duration of the attack prior to treatment.

The duration of follow-up and the last known acuity of the better seeing eye are shown in Table 2 .

\section{ANALYSIS OF VISUAL RESULTS}

Regression analyses have been carried out in order to determine the effects, if any, of age, duration, and the interaction of these 2 variables on visual acuity. A feature of this approach is that scores are assigned to the levels of visual acuity which were recorded. Regression makes several assumptions about the arithmetical properties of the scores once they have been assigned (e.g., that the difference in visual acuity between $6 / 6$ and $6 / 9$ is equal to the difference between $6 / 9$ and $6 / 18$ under system $A$ as defined below; that the difference between $6 / 6$ and $6 / 9$ is equal to that between $6 / 9$ and $6 / 12$ under system $B$ ). Alternative scoring systems were analysed in order to establish the robustness of the findings. It would not have been desirable that the substantial character of findings should be dependent merely on the scoring convention adopted. In the event fairly consistent

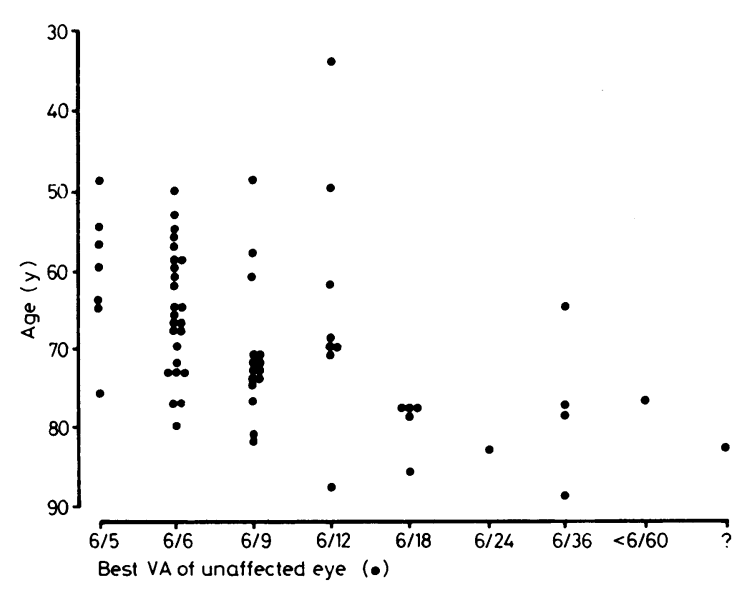

Fig. 3 Age of the patients and the best recorded acuity of the unaffected eye after prophylactic treatment.

findings emerge, albeit with some marginal differences of emphasis, irrespective of the scoring system used.

The scoring systems for visual acuity were:

$$
\begin{aligned}
& \text { System A: } \quad 6 / 5 \rightarrow 1 \cdot 2 \quad \text { System B: } \quad 6 / 5 \rightarrow 1 \\
& \begin{array}{ll}
6 / 6 \rightarrow 1 \cdot 0 & 6 / 6 \rightarrow 2 \\
6 / 9 \rightarrow 0 \cdot 67 & 6 / 9 \rightarrow 3
\end{array}
\end{aligned}
$$

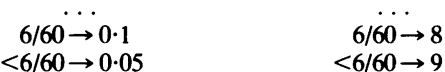

The regressions were carried out on:

(i) The best visual acuity recorded for the affected eye after surgery for the 71 cases recorded in Table 2, including both eyes in cases of bilateral attack.

(a) Visual acuity according to system $\mathrm{A}$ as a function of age and duration. The result was a significant $(<0.001)$ effect in age, but no further significant effect for either duration or interaction (age $\times$ duration) even at the lowest $(p<0.05)$ significance level. The equation is

$$
\text { Acuity (system A) = 1.531-0.015 age. }
$$

A considerable measure of individual patient variation remained unexplained, however, even when account was taken in this way of age (technically $80 \%$ of the variance remained unexplained).

(b) With acuity system B the result is broadly similar. It is, however, possible to demonstrate an effect attributed to duration in addition to that for age. The duration effect is just about significant at the $p=0.05$ level, while that for age is significant at $\mathrm{p}<0.001$. The equation obtained, which accounts for $21 \%$ of the variance, is

$$
\begin{aligned}
\text { Acuity (system B)= } & -2 \cdot 822 \\
& +0 \cdot 105 \text { age } \\
& +0 \cdot 057 \text { duration. }
\end{aligned}
$$


Table 1 Duration of stay in hospital

\begin{tabular}{|c|c|c|c|c|c|c|c|c|c|c|c|c|}
\hline \multirow[t]{2}{*}{ Days in hospital } & \multicolumn{12}{|c|}{ Year patient presented } \\
\hline & 1970 & 1971 & 1972 & 1973 & 1974 & 1975 & 1976 & 1977 & 1978 & 1979 & 1980 & Total \\
\hline 0 & - & - & - & - & - & - & 4 & 6 & 4 & 7 & 11 & 32 \\
\hline 1 & - & - & - & - & - & 4 & 6 & - & 4 & - & 1 & 15 \\
\hline 2 & - & - & - & 1 & 1 & 3 & - & - & - & - & - & 5 \\
\hline 3 & - & - & 1 & 2 & - & - & - & - & - & - & - & 3 \\
\hline 4 & - & 1 & 3 & 4 & 4 & - & - & - & - & - & - & 12 \\
\hline 5 & - & 2 & - & - & - & - & - & - & - & - & - & 2 \\
\hline 6 & - & 1 & - & - & - & - & - & - & - & - & - & 1 \\
\hline 7 & 1 & 1 & - & - & - & - & - & - & - & - & - & 2 \\
\hline \multicolumn{13}{|c|}{ Already in hospital } \\
\hline for other condition & - & - & - & - & 1 & 1 & - & - & 1 & - & - & 3 \\
\hline Total & 1 & 5 & 4 & 7 & 6 & 8 & 10 & 6 & 9 & 7 & 12 & 75 \\
\hline
\end{tabular}

This means that with system B acuity tends to be worse with increased duration when age is held constant, although the effect is a weak one.

(ii) The difference between the best recorded visual acuity of the affected eye and that of the other eye for the 62 patients (with complete data) who had prophylactic iridectomy.

(a) If the difference is calculated as simply scores under system $\mathrm{A}$ for affected eye minus the score for the other eye, it is found that neither age nor duration nor interaction contributes significantly to explaining the variation from patient to patient.

(b) If the difference is similarly calculated from scores with system B applied throughout, a weak yet significant $(p<0.05)$ effect in duration emerges:

\footnotetext{
Difference (system B) $=1 \cdot 158+0 \cdot 074$

duration accounts for $8 \%$ of the variance.
}

(iii) The best acuity recorded for the 'second' eyes after the 62 patients had prophylactic iridectomy. There was a statistically significant relationship between increasing age of the patient and worse visual acuity irrespective of the scoring system used. If the correlation coefficient, $r$, is calculated, it is found that

System A: $r=-0 \cdot 36, p=0 \cdot 0018$.
System B: $r=0 \cdot 37, p=0 \cdot 0015$

There was no significant association between the best visual acuity of these 'second' eyes and the duration of the symptoms affecting the 'first' eyes and/or the interaction of age and duration.

(iv) The data in Table 2 have also been examined to see if there is any correlation between the age of the patient and the duration of the symptoms. The coefficient of correlation, $r$, was calculated between the variables age and duration for the 68 patients concerned. The value obtained $r=-0.060$ was not significant at the $p<0.05$ level.
Conclusion. There was no evidence in this series to suggest that the aged delayed seeking attention.

\section{Discussion}

\section{MANAGEMENT OF THE PATIENT}

The progressive reduction in duration of hospitalisation after ocular surgery was not anticipated in 1970, but it has been reflected in this series (Table 1). While this was taking place, attention became concentrated less on the affected eye and rather more on the patients and their reaction to acute glaucoma and the treatment. When a number of them had been treated without admission to hospital, it was evident that they were not, generally speaking, 'ill,' and there were none of the medical and social problems associated with hospitalisation. They appeared to be coming through a potentially serious sightthreatening episode with remarkably little concern. This seemed to be due to 2 factors. Firstly, they had only one visit to the operating theatre, and, secondly, strong reassurance (fully justified by these results) that they would not go blind from glaucoma now or in the future. Their confidence was reinforced by being told that, although they required a preventive operation on their unaffected eye, it was not really necessary for them to come into hospital, and they could have it done at the same time as the affected eye was operated upon.

Unfortunately it was not always possible to achieve the ultimate in speed and simplicity. For example, retrobulbar haemorrhage required a second visit to the theatre for 5 patients, and one required a general anaesthetic. Nevertheless a policy of confident reassurance, one (bilateral) operation, and no hospitalisation appears to have minimised morbidity and the need for rehabilitation.

RESULTS OF TREATING ACUTE GLAUCOMA

Recovery of vision in an eye affected by acute 
Table 2 Last known visual acuity of the better seeing eye and period of follow-up

\begin{tabular}{|c|c|c|c|c|c|c|c|c|c|c|}
\hline \multirow{2}{*}{$\begin{array}{l}\text { Duration of } \\
\text { follow-up } \\
\text { in years }\end{array}$} & \multicolumn{10}{|c|}{ Visual acuity } \\
\hline & $6 / 5$ & $6 / 6$ & $6 / 9$ & $6 / 12$ & $6 / 18$ & $6 / 24$ & $6 / 36$ & $6 / 60$ or less & $?$ & Total \\
\hline$<1$ & - & 2 & 3 & 1 & 1 & 1 & - & 2 & 1 & 11 \\
\hline $1+$ & 1 & 5 & 3 & 1 & - & - & 1 & - & - & 11 \\
\hline $2+$ & 3 & 4 & 2 & - & 2 & - & 1 & - & - & 12 \\
\hline $3+$ & 1 & 2 & 2 & 2 & 1 & 1 & - & - & - & 9 \\
\hline $4+$ & 1 & 6 & 1 & 1 & 1 & - & - & - & - & 10 \\
\hline $5+$ & - & 4 & 3 & 1 & 1 & - & - & 1 & - & 10 \\
\hline $6+$ & - & 1 & 1 & - & - & - & - & - & - & 2 \\
\hline $7+$ & - & - & 1 & 1 & - & - & - & - & - & 2 \\
\hline $8+$ & - & - & 2 & 1 & - & - & 1 & - & - & 4 \\
\hline $9+$ & 2 & 1 & - & - & - & - & - & - & - & 3 \\
\hline $10+$ & - & 1 & - & - & - & - & - & - & - & 1 \\
\hline Total & 8 & 26 & 18 & 8 & 6 & 2 & 3 & 3 & 1 & 75 \\
\hline
\end{tabular}

Of the 8 patients whose last known visual acuity was $6 / 24$ or less, 1 had cataracts, 3 had senile macular degeneration, 1 had diabetic maculopathy, 1 had vitreous haemorrhages from hypertensive retinopathy, and 1 had acute glaucoma in an only eye; 1 was aged 90 years.

Sixty-nine patients had unilateral acute glaucoma and a prophylactic iridectomy on the fellow eye. In 48 of these (70\%) the eye that had prophylactic surgery had the better vision when last seen, and in $8(11.6 \%)$ the reverse was true. In 13 others the acuity of the 2 eyes was equal.

Three patients have had bilateral cataract extraction since their acute glaucoma.

glaucoma is variable. Pressure remained raised in 7 eyes $(9 \%)$ after the attack. The remainder settled, but it was often several months before the best acuity was obtained, ${ }^{2}$ as is the case of other intraocular operations.

It is commonly believed that delay in starting treatment for acute glaucoma causes more sight to be lost. However, it is important to differentiate between vision 'lost' and the vision recovered or retained after the attack, which is what previous authors have in fact recorded. An association between the duration of the attack and this, the vision retained, has not been confirmed, ${ }^{34}$ and it is not obvious in this series either (see Fig. 1), although it can just be demonstrated statistically by one of 2 possible methods of scoring visions.

So far as we are aware the patient's age has not been considered relevant to the visual outcome. Yet this is clearly an important factor, as older people retained significantly less vision than younger ones. The aged were not more prone to delay seeking attention nor were they more at risk if they delayed treatment (there being no demonstrable interaction between, on the one hand, the patient's age and the duration of the attack and, on the other hand, the vision recovered).

It is seldom possible to know the acuity of an eye immediately before an attack of acute glaucoma, but one way to explore factors associated with visual loss caused by acute glaucoma would be to compare the vision of the affected eye with that of the unaffected eye after both had been treated with miotics and iridectomy. The literature suggests that prophylactic iridectomy seldom causes the loss of acuity of more than one line on the Snellen chart. ${ }^{56}$ It is possible therefore to suppose that acuity recovered after prophylactic treatment reflects acuity before treatment. Moreover, in the absence of unilateral pathology, visual acuity of a pair of eyes is roughly equal. If it is permissible to make these assumptions, there is only a minimal suggestion that increased duration of the attack caused more sight to be lost. An increased difference between the vision of the 2 eyes after treatment could only be related to the duration of the attack when one of the 2 methods of scoring vision was used. The difference was not more marked in the elderly. On the other hand, the vision recovered after the originally unaffected eye had been treated was clearly age-dependent, as was the case with the affected eye. Although one cannot eliminate the possibility that the aged patient's eyes were more susceptible to the effects of miotics and iridectomy, the likely explanation for the age dependency of vision recovered after prophylactic treatment is that, in the case of the aged, acuity was lower to start with (unfortunately pretreatment acuity of the unaffected eye was not recorded often enough to confirm this). By the same argument one could also postulate that the reason why the aged recovered less vision after acute glaucoma was that they had less vision to start with. The relatively consistent difference, which is not age-dependent, between the vision of the affected and unaffected eyes after treatment obviously confirms that the attack itself was a prime cause of loss of vision.

\section{LONG-TERM PROGNOSIS}

As regards the vision of the individual patients, the long-term prognosis for sight in at least one eye appears to be satisfactory (Table 2). Only one 
patient, a woman of 83 years who had acute glaucoma in an only eye, was blinded by the attack. Four of the affected eyes that recovered vision of better than $6 / 60$ have had raised intraocular pressure, but only one of them subsequently ( 9 years after the attack) developed glaucomatous cupping and loss of vision. Three of the eyes that had prophylactic iridectomy have ocular hypertension, and, although one of them has developed a shallow atrophic cupping of the disc in spite of normal pressure, none have lost sight from chronic glaucoma.

When last seen, $11 \%$ of those patients who had unilateral acute glaucoma had a lower acuity in the eye that had prophylactic iridectomy, and this suggests that prophylactic iridectomy is not ideal. Mapstone $^{7}$ is surely right, therefore, to question the indiscriminate use of prophylactic iridectomy. On the other hand the originally unaffected eye was the better seeing eye in $70 \%$ of patients despite prophylactic treatment. One can only speculate on how many of these patients would have by now had acute glaucoma in their second eye if they had not had prophylactic iridectomy. If nothing else, confident reassurance that prophylactic surgery insures against sudden loss of sight from acute glaucoma should reduce morbidity.

It is not the purpose of this paper to discuss the advantages and disadvantages of prophylactic surgery. However, more people are living into the $70+$ age group, and it is right to stress the point that, as the age of the individual increases, the chances of recovering vision after acute glaucoma in an unprotected eye decrease. If a policy of prophylactic iridectomy is accepted, the operation might as well be done soon, and there appears to be no reason why it cannot be done simultaneously with surgery for the affected eye. Indeed, this is exactly the advice given in the most recent publication ${ }^{8}$ on the subject.

\section{References}

1 Lowe RF. Primary angle-closure glaucoma. Problems of prevention. Trans Ophthalmol Soc UK 1970; 90: 323-7.

2 McNaught EI, Rennie A, McClure E, Chisholm IA. Pattern of visual damage after acute angle-closure glaucoma. Trans Ophthalmol Soc UK 1974; 94: 406-15.

3 Mackie EG, Rubinstein K. Iridencleisis in congestive glaucoma. Br J Ophthalmol 1954; 38: 641-52.

4 Hillman JS. Acute closed-angle glaucoma: an investigation into the effect of delay in treatment. BrJ Ophthalmol 1979; 63: 817-21.

5 Ghoshal TK, Blaxter PL. Results of peripheral iridectomy in closed-angle glaucoma. Br J Ophthalmol 1969; 53: 110-5.

6 Douglas WHG, Strachan IM. Surgical safety of prophylactic peripheral iridectomy. Br J Ophthalmol 1967; 51: 459-62.

7 Mapstone R. The fellow eye. Br J Ophthalmol 1981; 65: 410-3.

8 Edwards RS. Behaviour of the fellow eye in acute angle-closure glaucoma. Br J Ophthalmol 1982; 66: 576-9. 